International Journal of Physical Research, $7(1)(2019) 7-10$
International Journal of Physical Research
Website $:$ www.sciencepubco.com/index.php/IJPR
Research paper

\title{
EOS parameters and elastic properties of cubic rock-salt BP
}

\author{
Salah Daoud * \\ Laboratory of Materials and Electronic Systems (LMSE), Faculty of Sciences and Technology, \\ Mohamed Elbachir El Ibrahimi, Bordj Bou Arreridj University, 34000 Bordj Bou Arreridj, Algeria \\ *Corresponding author E-mail: salah_daoud07@yahoo.fr
}

\begin{abstract}
The present work aims to study the equation of state (EOS) under compression up to $100 \mathrm{GPa}$, and the elastic properties of cubic rocksalt Boron phosphide (BP) material. The EOS parameters and the elastic constants of our material of interest were predicted using plane wave-pseudopotential (PW-PP) approach in the framework of the density functional theory (DFT) and the density functional perturbation theory (DFPT) with the generalized gradient approximation (GGA) for the exchange-correlation functional. The Young modulus, the Poisson's ratio, the sound velocity, the Debye temperature and the melting temperature of the aggregate material were also presented. The results obtained are in general in good agreement compared to other data of the literature.
\end{abstract}

Keywords: Ab-Initio Calculation; BP Material; Equation of State; Elastic Constants; Thermal Properties.

\section{Introduction}

Because of the good performance of boron phosphide (BP) semiconducting compound, such as high melting points, high hardness, dielectric and thermal characteristics as well as its unusual behavior, it attracts more attention in recent years [1]. Using the linear muffin-tin orbitals (LMTO) approach and the pseudopotential - plane waves (PP-PW) method, the structural and electronic properties of BP with three different structures (cubic zinc-blende, $\beta$-Sn and cubic rock-salt phases) were systematically investigated by Kocinski and Zbroszczyk [1]. Theirs calculations indicate that the boron phosphide material crystallizes in the cubic zinc-blende phase, and it has an indirect gap of approximately $1.5 \mathrm{eV}$, which decreases quadratically under compression. They found also that the phase transition from zinc-blende to rock-salt phase occurs at extremely high pressure (approximately $150 \mathrm{GPa}$ ).

Using first principles total-energy calculations based on the full-potential augmented plane-wave plus local orbitals (FP-LAPW+lo) method, Bouhemadou et al. [2] have studied the effect of the high pressure on the structural and mechanical properties of some IIIphosphide compounds (BP, GaP, AlP, InP). They found a linear dependence between the elastic constants and the applied pressure. They calculated also the sound velocity and the Debye temperature $\theta_{\mathrm{D}}$ of the aggregate materials.

Using the same approach, Arbouche et al. [3] have studied the effect of high pressure on the phase transition of the same materials (BP, $\mathrm{GaP}$, AlP, InP). For BP material, they found that this compound transforms from the zinc-blende structure to the rock-salt phase at around $133 \mathrm{GPa}$, then from the rock-salt phase to the NiAs phase at a pressure of around $212 \mathrm{GPa}$.

In our previous work [4-6], the elastic constants, the piezoelectric coefficients, the electronic properties and some other physical properties of zincblende BP material under high pressure were studied using ab-initio calculations. We found that the elastic constants increase monotonously with increasing pressure, while the piezoelectric coefficients decrease with the increase of pressure.

Although the cubic rock-salt phase of boron phosphide material is not synthesized until now, we will test to extent our study on the cubic rock-salt phase of this compound, so some other properties of this phase have been systematically studied here. In the present work, we compute the structural parameters, the elastic constants and some other physical quantities of BP material in cubic rock-salt phase.

\section{Computational method}

The structural parameters and the elastic constants calculated in the present study were carried out using ABINIT code [7], which based on the PP-PW approach in the framework of the DFT and the DFPT. To describe the interactions between the valence electrons and the nuclei and core electrons, the Troullier-Martins type pseudopotentials which have been generated thanks to the FHI98PP code [8] were employed. The exchange-correlation energy was evaluated in the generalized gradient approximation (GGA) [9]. The total energy was found converged near the energy cutoff of $80 \mathrm{Ha}$. The Brillouin zone was sampled by $(12 \times 12 \times 12)$ Monkhorst and Pack mesh of $\mathrm{k}$ points [10]. For more detail on the calculation of the structural parameters, and the elastic constants of materials, please see for example our previous works [4-6]. 


\section{Results and discussion}

\section{1. EOS parameters and crystal density}

To predict the equilibrium structural parameters of cubic rock-salt BP, the pressure-volume $(\mathrm{P}-\mathrm{V})$ data was employed. The unit cell volumes at fixed values of applied pressure were used to construct the equation of state (EOS). The normalized lattice parameter (ap/an) versus pressure in the range from 0 to $100 \mathrm{GPa}$ for cubic rock-salt BP was plotted in Fig. 1, along with the theoretical one reported by Arbouche et al. [3]

From figure 1, we observe that the normalized lattice parameter $\left(\mathrm{a}_{\mathrm{p}} / \mathrm{a}_{0}\right)$ of our material of interest decreases with the increase of pressure, where it is started with the value 1 at zero-pressure, and it reaches the value 0.896 at $100 \mathrm{GPa}$.

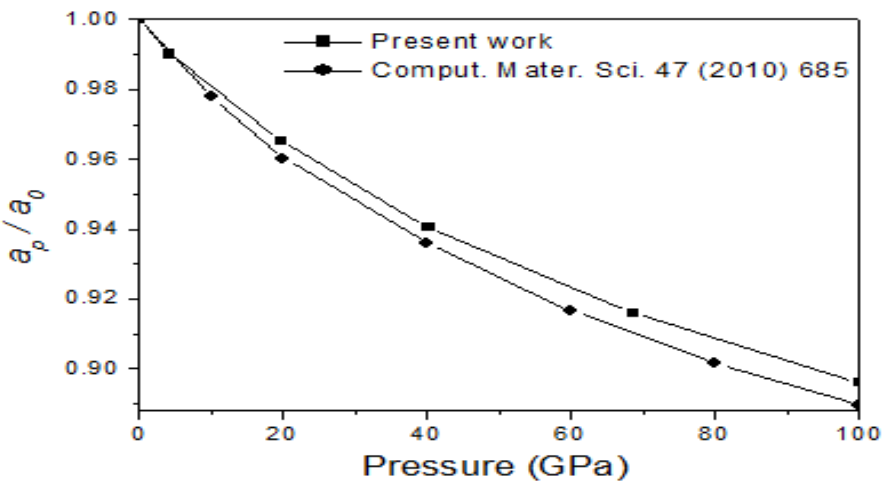

Fig. 1: Normalized Lattice Parameter $\left(\mathrm{a}_{\mathrm{p}} / \mathrm{a}_{0}\right)$ Versus Pressure.

The calculated normalized volume $\left(\mathrm{V}_{\mathrm{p}} / \mathrm{V}_{0}\right)$ versus pressure is plotted in Fig. 2, along with the theoretical one reported by Arbouche et al. [3]. From figure 2, we observe that $\mathrm{V}_{\mathrm{p}} / \mathrm{V}_{0}$ of $\mathrm{BP}$ decreases also with increasing pressure, where it is started with the value 1 at zero pressure, and it reaches the value 0.716 at $100 \mathrm{GPa}$.

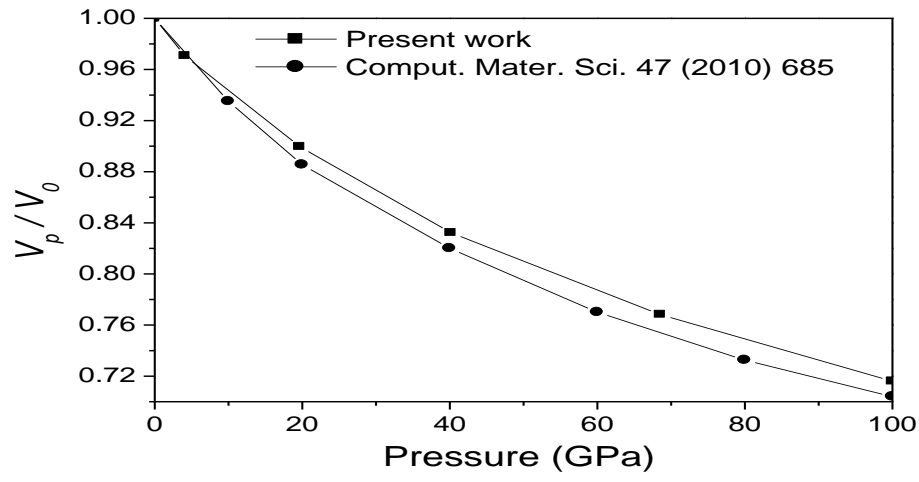

Fig. 2: Normalized Volume $\left(\mathrm{V}_{\mathrm{p}} / \mathrm{V}_{0}\right)$ Versus Pressure.

The EOS parameters (equilibrium lattice constant a , bulk modulus $\mathrm{B}_{0}$, and its pressure derivatives $\mathrm{B}_{0}$ ) can be determined through the well-known pressure-normalized volume $\left(\mathrm{P}-\left(\mathrm{V} / \mathrm{V}_{0}\right)\right)$ third-order Vinet EOS, which is given as follow [11], [12]

$P(V)=3 B_{o}\left[\frac{1-\left(V / V_{o}\right)^{1 / 3}}{\left(V / V_{0}\right)^{2 / 3}}\right] \exp \left\{\frac{3}{2}\left(B_{o}{ }^{\prime}-1\right)\left[1-\left(V / V_{o}\right)^{1 / 3}\right]\right\}$

Where: $\mathrm{V}$ is the volume at pressure $\mathrm{P}$, and $\mathrm{V}_{0}$ is the volume at zero-pressure, respectively.

The calculated data $\left(\mathrm{P}-\left(\mathrm{V} / \mathrm{V}_{0}\right)\right)$ of BP material with $\mathrm{B} 1$ structure was plotted in Fig. 3.

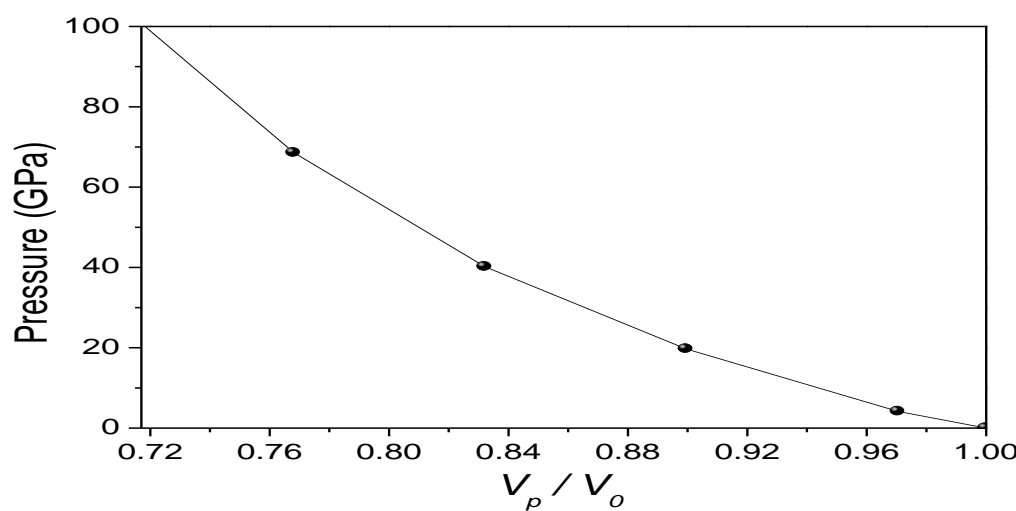

Fig. 3: Pressure versus $\left(\mathrm{V}_{\mathrm{p}} / \mathrm{V}_{0}\right)$ of $\mathrm{BP}$ with $\mathrm{B} 1$ Phase. 
The obtained value of $\mathrm{a}_{0}, \mathrm{~B}_{0}$, and $\mathrm{B}_{0}{ }^{\prime}$ are given in Table 1, and compared with other theoretical data [1], [3] and [13] of the literature. In general, our calculated values of $\mathrm{a}_{0}, \mathrm{~B}_{0}$ and $\mathrm{B}_{0}{ }^{\prime}$ are agreed with other theoretical data [1], [3] and [13].

Table 1: Equilibrium Lattice Constant $\mathrm{a}_{0}$, Bulk Modulus $\mathrm{B}_{0}$, and Its Pressure Derivatives $\mathrm{B}_{0}{ }^{\prime}$ of BP with Rock-Salt Structure, Compared to other Theoretical Data of The Literature. ${ }^{\mathrm{a}} \mathrm{PP}-\mathrm{PW},{ }^{\mathrm{b}} \mathrm{LMTO},{ }^{\mathrm{c}} \mathrm{LDA},{ }^{\mathrm{d}} \mathrm{GGA}$

\begin{tabular}{llll}
\hline Parameter & $\mathrm{a}_{0}(\AA)$ & $\mathrm{B}_{0}(\mathrm{GPa})$ & $\mathrm{B}_{0}{ }^{\prime}$ \\
\hline This work & 4.282 & 148.4 & 152 \\
Ref. [1] & 4.30 & 176 & - \\
Ref. [1] & & & \\
Ref. [3] & 4.27 & 163.2 & 3.64 \\
Ref. [13] & 4.32 & 177 & 3.32 \\
Ref. [13] ${ }^{\mathrm{d}}$ & 4.268 & 161 & 3.60 \\
\hline
\end{tabular}

The deviation between our calculation (148.4 GPa) of $\mathrm{B}_{0}$ and the theoretical one (152 GPa) reported by Kocinski and Zbroszczyk [1] is only about $2.4 \%$. It can be seen that our value (4.64) of $\mathrm{B}_{0}{ }^{\prime}$ is slightly higher than the previous results 3.58 and 3.32 (3.6) reported by Arbouche et al. [3], and Meradji et al. [13], respectively.

We further investigate the high-pressure crystal density g of our compound of interest. The calculated crystal density at different values of pressure is plotted in Fig. 4. An analytical relation for the pressure dependence of the crystal density is given by the following a quadratic fit:

$$
\mathrm{g}=3.554+1.932 \times 10^{-2} \mathrm{p}-0.55 \times 10^{-4} \mathrm{p}^{2}
$$

Where $\mathrm{p}$ is given in $\mathrm{GPa}$, and $\mathrm{g}$ in $\mathrm{g} / \mathrm{cm}^{3}$.

At zero-pressure, our calculated value of the crystal density $\mathrm{g}$ was found at around $3.534 \mathrm{~g} / \mathrm{cm}^{3}$.

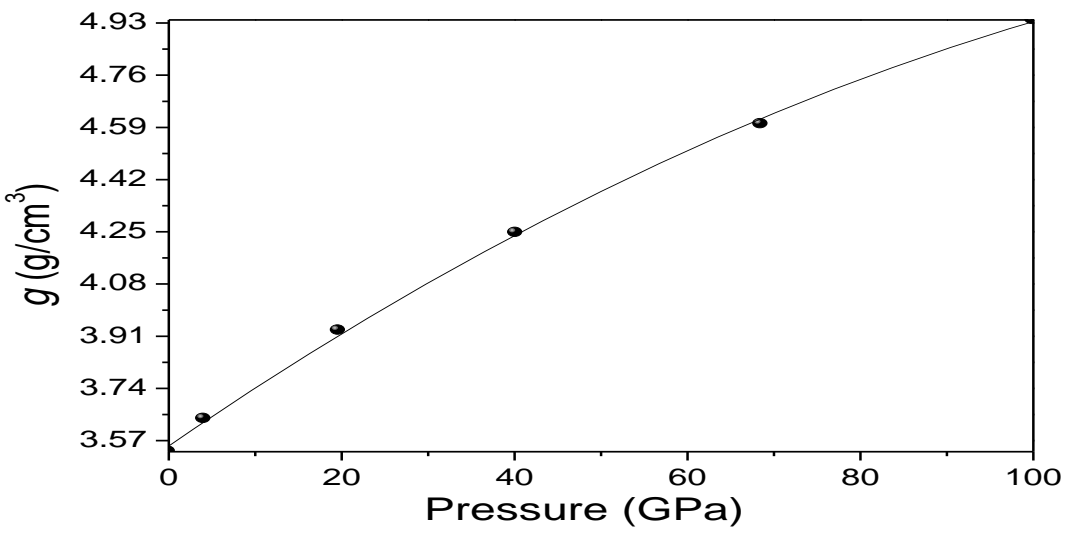

Fig. 4: Crystal density Versus Pressure of BP Up to $100 \mathrm{GPa}$.

\subsection{Elastic constants, young modulus and poisson's ratio}

The elastic constants obtained here were calculated by the density functional perturbation theory (DFPT) method. For more detail on the calculation of the elastic constants of materials by the DFPT, please see for example our previous works [6, 14]. The obtained value of $\mathrm{C}_{11}, \mathrm{C}_{12}$ and $\mathrm{C}_{44}$ for BP material with B1 structure are given in Table 2, and compared with other theoretical data [15].

Table 2: Elastic Constants $\mathrm{C}_{\mathrm{ii}}$ (in GPa) of BP with Rock-Salt Structure, Compared to Other Theoretical Data [15]

\begin{tabular}{llll}
\hline Parameter & $\mathrm{C}_{11}$ & $\mathrm{C}_{12}$ & $\mathrm{C}_{44}$ \\
\hline This work & 326.4 & 68.2 & 274.9 \\
Ref. [15] & 341 & 86 & 248 \\
\hline
\end{tabular}

From the Table 2, it is observed that our obtained values of $\mathrm{C}_{11}, \mathrm{C}_{12}$, and $\mathrm{C}_{44}$ are in general in agreement compared to other theoretical ones [15]. Our value (326.4 GPa) of $\mathrm{C}_{11}$ underestimate the theoretical one (326.4 GPa) [15] obtained from the full potential linearized augmented plane wave (FP-LAPW) method by only about $4.3 \%$. To the best of our knowledge, there are no other data (except the work [15]) available in the literature on the elastic constants for BP compound with rock-salt structure.

For the aggregate polycrystalline materials, the average quantities of the mechanical properties are usually used. The Young modulus $\mathrm{E}$ and the Poisson's ratio $\sigma$ are usually expressed as function of the bulk modulus B and the isotropic shear modulus G as follow [16]

$\mathrm{E}=9 \mathrm{BG} /(3 \mathrm{~B}+\mathrm{G})$, and $\sigma=(3 \mathrm{~B}-2 \mathrm{G}) /(6 \mathrm{~B}+2 \mathrm{G})$

Our calculated values of B, G, E and $\sigma$ of BP aggregate polycrystalline material are: 154.25 GPa, 202.97 GPa, 423.26 GPa and 0.04, respectively. Our calculated value $(154.25 \mathrm{GPa})$ of B agreed well with the value $(148.4 \mathrm{GPa})$ obtained previously in the present work from the P-V data. It is also in good agreement with the theoretical result (152 GPa) reported by Kocinski and Zbroszczyk [1]. The deviation between these two values is only around $1.5 \%$.

\subsection{Sound velocity, Debye temperature and melting temperature}

The longitudinal $\mathrm{v}_{\mathrm{l}}$, transverse $\mathrm{v}_{\mathrm{t}}$, and average $\mathrm{v}_{\mathrm{m}}$ sound velocities as well as the Debye temperature are calculated, the details are described elsewhere [17-20]. The calculated values of $\mathrm{vl}, \mathrm{vt}$ and $\mathrm{vm}$ of BP compound with B1 structure are: $10.965,7.579$ and $8.244 \mathrm{~km} / \mathrm{s}$, respectively. The calculated value of the Debye temperature $\theta_{D}$ of BP with rock-salt structure is around $1147 \mathrm{~K}$. This value is slightly 
higher than the value $1095 \mathrm{~K}$ of the zinc-blende phase reported by Bouhemadou et al. [2]. For crystals with cubic structure symmetry, the melting temperature $T_{m}$ and the elastic constant $C_{11}$ are related by [5], [16]

$\mathrm{T}_{\mathrm{m}}=553+(591 / \mathrm{Mbar}) \mathrm{C}_{11} \pm 300 \mathrm{~K}$

The value of $\mathrm{T}_{\mathrm{m}}$ for BP with B1 structure has been found $2482 \pm 300 \mathrm{~K}$. To the best of our knowledge, there are no other data available in the literature on the sound velocity and the melting temperature $\mathrm{T}_{\mathrm{m}}$ for $\mathrm{BP}$ compound with $\mathrm{B} 1$ structure.

\section{Conclusion}

Using PW-PP approach in the framework of the DFT and the DFPT, we determine the EOS parameters of cubic rock-salt BP material using the pressure-volume data. The values obtained are in general in agreement with other theoretical ones of the literature.

The pressure dependence of the crystal density under high pressure up to $100 \mathrm{GPa}$ was also studied.

The elastic constants, the bulk modulus, the isotropic shear modulus, the Young modulus and the Poisson's ratio are also predicted. In addition, the longitudinal, transverse and average elastic wave velocities and also the Debye temperature $\theta_{\mathrm{D}}$ of the aggregate material were also presented. It was found that the Debye temperature $\theta_{\mathrm{D}}$ of the cubic rock-salt structure is slightly higher than the value of the cubic zinc-blende phase reported in the literature.

To the best of our knowledge, there are no data available in the literature on the longitudinal, transverse and average elastic wave velocities, and the Debye temperature $\theta_{\mathrm{D}}$ for BP compound with B1 structure. So, future experimental and theoretical results are needed to valid our finding data of B1 phase.

\section{References}

[1] P. Kocinski and M. Zbroszczyk, "Calculated structural and electronic properties of boron phosphide under pressure", Semiconductor Science and Technology, Vol. 10, No. 11, (1995), pp. 1452-1457. https://doi.org/10.1088/0268-1242/10/11/004.

[2] A. Bouhemadou, R. Khenata, M. Kharoubi, T. Seddik, Ali H. Reshak, and Y. Al-Douri, " FP-APW + lo calculations of the elastic properties in zinc-blende III-P compounds under pressure effects ", Computational Materials Science, Vol. 45, No. 2, (2009), pp. 474-479. https://doi.org/10.1016/j.commatsci.2008.11.013.

[3] O. Arbouche, B. Belgoumène, B. Soudini, Y. Azzaz, H. Bendaoud, and K. Amara, " First-principles study on structural properties and phase stability of III-phosphide (BP, GaP, AlP and InP) ", Computational Materials Science, Vol. 47, No. 3, (2010), pp. 685-692. https://doi.org/10.1016/j.commatsci.2009.10.009.

[4] S. Daoud, K. Loucif, N. Bioud, N. Lebga, and L. Belagraa, " Effect of hydrostatic pressure on the structural, elastic and electronic properties of (B3) boron phosphide ", Pramana Journal of Physics, Vol. 79, No. 1, (2012), pp. 95-106. https://doi.org/10.1007/s12043-012-0283-8.

[5] S. Daoud, N. Bioud, and N. Lebga, "Elastic, optoelectronic and thermal properties of boron phosphide ", Journal of Nano- and Electronic Physics, Vol. 5, No. 4, (2013), pp. 04061 (6 pages). https://jnep.sumdu.edu.ua/download/numbers/2013/4/articles/jnep_2013_V5_04061.pdf.

[6] S. Daoud, N. Bioud, and N. Lebga, "Mechanical, piezoelectric and some thermal properties of (B3) BP under pressure", Journal of Central South University, Vol. 21, No. 1, (2014), pp. 58-64. https://doi.org/10.1007/s11771-014-2394-4.

[7] The ABINIT code is a common project of the Université Catholique de Louvain, Corning Incorporated, and other contributors. http://www.abinit.org.

[8] M. Fuchs and M. Scheffler, "Ab initio pseudopotentials for electronic structure calculations of poly-atomic systems using density- functional theory", Computer Physics Communications, Vol.119, No. 1, (1999), pp. 67-98. https://doi.org/10.1016/S0010-4655(98)00201-X.

[9] J.P. Perdew, K. Burke, and M. Ernzerhof, "Generalized gradient approximation made simple", Physical Review Letters, Vol. 77, No. 18, (1996), pp. 3865 - 3868. https://doi.org/10.1103/PhysRevLett.77.3865.

[10] H.J. Monkhorst, and J.D. Pack, "Special points for Brillouin-zone integrations", Physical Review B, Vol. 13, No. 12, (1976), pp. 5188 - 5192. https://doi.org/10.1103/PhysRevB.13.5188.

[11] S. Daoud, R. Mezouar and A. Benmakhlouf, " Equation of state and some other properties of rock-salt AlN ", International Journal of Physical Research, Vol. 6, No. 1, (2018), pp. 49-52. https://doi.org/10.14419/ijpr.v6i1.10754.

[12] S. Daoud, R. Mezouar, and A. Benmakhlouf, " Structural and piezoelectric coefficients of AlP under pressure", International Journal of Physical Research, Vol. 6, No. 2, (2018), pp. 53-55. https://doi.org/10.14419/ijpr.v6i2.11020.

[13] H. Meradji, S. Drablia, S. Ghemid, H. Belkhir, B. Bouhafs \& A. Tadjer, "First-principles elastic constants and electronic structure of BP, BAs, and BSb", Physica Status Solidi B, Vol. 241, No. 13, (2004) pp. 2881-2885. https://doi.org/10.1002/pssb.200302064

[14] S. Daoud, "Mechanical and piezoelectric properties, sound velocity and Debye temperature of thallium-phosphide under pressure", International Journal of Advanced Research in Physical Science, Vol. 1, No. 6, (2014), pp. 1-11. www.arcjournals.org/pdfs/ijarps/v1i6/1.pdf.

[15] F. El Haj Hassan, H. Akbarzadeh, and M. Zoaeter, "Structural properties of boron compounds at high pressure", Journal of Physics: Condensed Matter, Vol. 4, No. 3, (2004), pp. 293-301. https://doi.org/10.1088/0953-8984/16/3/009.

[16] S. Daoud, N. Bioud, and N. Lebga, " Elastic and thermophysical properties of BAs under high pressure and temperature ", Chinese Journal of Physics, Vol. 57, (2019), pp. 165-178. https://doi.org/10.1016/j.cjph.2018.11.018.

[17] S. Daoud, " Sound velocities and thermal properties of BX (X=As, Sb) compounds", International Journal of Scientific World: Vol. 3, No. 1 (2015), pp. 43-48. https://doi.org/10.14419/ijsw.v3i1.4039.

[18] S. Daoud, " Sound velocities and thermal properties of BeX (X=S, Se and Te) alkaline-earth chalcogenides ", International Journal of Scientific World: Vol. 5, No. 1 (2017), pp. 9-12. https://doi.org/10.14419/ijsw.v5i1.6929.

[19] S. Daoud, and N. Bouarissa, "Elastic, piezoelectric and thermal properties of zinc-blende AlN under pressure ", Theoretical Chemistry Accounts, Vol. 138, No. 4, (2019), pp. 49 (10 pages). https://doi.org/10.1007/s00214-019-2439-9.

[20] S.U. Rehman, F.K. Butt, F. Hayat, B. Ul Haq, Z. Tariq, F. Aleem, and C. Li, "An insight into a novel cubic phase SnSe for prospective applications in optoelectronics and clean energy devices", Journal of Alloys and Compounds, Vol. 733, No. 5 (2018), pp. 22-32. https://doi.org/10.1016/j.jallcom.2017.10.192. 